\title{
Fadiga pré-operatória em pacientes com câncer: prevalência e fatores associados
}

\author{
Murielly Marques de Oliveira ${ }^{1}$, \\ Dálete Delalibera Corrêa de Faria Mota², \\ Juliana Nery de Souza-Talarico ${ }^{3}$
}

\section{RESUMO}

Este estudo objetivou descrever a prevalência de fadiga e identificar os fatores associados à fadiga em pacientes no pré-operatório de cirurgia oncológica. Pesquisa transversal, desenvolvida com 117 pacientes com câncer em pré-operatório (idade média= 51,2 anos; 76,9\% mulheres; 65,8\% viviam com companheiro; 70,9\% não realizaram tratamento prévio para câncer). Instrumentos utilizados foram Escala de Fadiga de Piper - Revisada; Escala de Estresse Percebido; Escala Hospitalar de Ansiedade e Depressão; Escala Numérica de Dor; Escala Numérica de Sono e Escala de Karnofsky (KPS). Realizou-se teste de qui-quadrado, teste t-Student, ANOVA oneway. Constatamos que a prevalência da fadiga pré-operatória foi de $25,6 \%$ e a intensidade foi moderada/intensa. A dimensão afetiva de fadiga apresentou escore mais alto comparado às dimensões comportamental e sensorial/psicológica. Ansiedade, depressão, estresse, dor, sono e performance status se associaram à fadiga préoperatória.

Descritores: Fadiga; Neoplasias; Procedimentos Cirúrgicos Operatórios; Enfermagem Cirúrgica.

\footnotetext{
${ }^{1}$ Enfermeira, Mestre em Enfermagem. Discente do Programa de Pós-Graduação em Enfermagem, nível Doutorado, da Universidade Federal de Goiás. Goiânia, GO, Brasil. E-mail: muriellymarques@gmail.com.

2 Enfermeira, Doutora em Enfermagem. Professora Associada da University of Wisconsin - Eau Claire. Eau Claire, WI, EUA. E-mail: dalete.mota@gmail.com.

${ }^{3}$ Enfermeira, Doutorado em Enfermagem na Saúde do Adulto e do Idoso. Professora Doutora da Escola de Enfermagem da Universidade de São Paulo. São Paulo, SP, Brasil. E-mail: junery@usp.br.
}

Artigo recebido: 02/12/2016.

Artigo aprovado: 20/02/2018.

Artigo publicado: 05/09/2018.

\section{Como citar esse artigo:}

Oliveira MM, Mota DDCF, Souza-Talarico JN. Fadiga pré-operatória em pacientes com câncer: prevalência e fatores associados. Rev. Eletr. Enf. [Internet]. 2018 [acesso em: ];20:v20a17. Disponível em: https://doi.org/10.5216/ree.v20.44440. 


\section{INTRODUÇÃO}

A fadiga do paciente cirúrgico ainda é pouco estudada e dados sobre esse tema no pré-operatório são incipientes ${ }^{(1-2)}$. Pacientes fatigados no pré-operatório tendem a ficar mais fatigados no pós-operatório e, consequentemente, podem apresentar recuperação pós-operatória mais lenta, prolongando o período de hospitalização, aumentando o risco de aquisição de infecção hospitalar, interferindo na continuidade do tratamento anticâncer, postergando o início de terapias adjuvantes ${ }^{(1-3)}$. Assim, realizar uma avaliação desse sintoma no pré-operatório se torna útil no planejamento de intervenções individualizadas, como organização do ciclo atividade/repouso, orientação nutricional e manejo de possíveis fatores causais como depressão ou anemia $^{(4)}$.

Fadiga é um sintoma subjetivo, multidimensional, multifatorial. É uma sensação física desagradável, com sintomas cognitivos e emocionais descritos como cansaço, e que não é aliviada com a aplicação de estratégias usuais de restauração e preservação de energia(5). Pacientes fatigados expressam sentimentos de cansaço, incapacidade de manter a rotina usual, perda de libido, verbalização de uma constante falta de energia, entre outros $^{(6)}$.

A fadiga pré-operatória raramente é um sintoma isolado, ocorrendo mais comumente associada a outros sintomas. Em revisão integrativa que buscou descrever a evolução de fadiga perioperatória em pacientes com câncer e seus fatores associados, a fadiga pré-operatória apresentou-se, frequentemente, associada à ansiedade e depressão. Também permitiu observar que a sua intensidade tendeu a aumentar em pacientes submetidos à quimioterapia ou radioterapia neoadjuvantes, atingindo um nível moderado ${ }^{(7-9)}$.

Existe a necessidade de pesquisa referente à fadiga em pacientes oncológicos submetidos à cirurgia, uma vez que a fadiga pré-operatória é uma experiência que tem repercussão negativa significativa. Percebemos que há lacuna de conhecimento sobre essa experiência multidimensional no Brasil, sobre os fatores relacionados à sua ocorrência e intensidade, dificultando o planejamento de ações mais efetivas para prevenção e tratamento do sintoma por parte dos profissionais de saúde. Sabendo disso, desenvolveu-se a presente pesquisa que buscou descrever a prevalência de fadiga e identificar os fatores associados à presença da fadiga em pacientes oncológicos no período pré-operatório.

\section{MÉTODO}

Trata-se de estudo transversal analítico, com amostra de conveniência constituída por 117 indivíduos adultos, com indicação de cirurgia oncológica convencional e eletiva, realizada entre abril de 2014 e janeiro de 2015, em duas instituições hospitalares de grande porte do município de Goiânia, Goiás, Brasil.

Os critérios de inclusão foram: ter idade maior ou igual a 18 anos, ter indicação de cirurgia para tratamento do câncer com finalidade curativa ou paliativa, de médio ou grande porte; apresentar avaliação de risco cirúrgico, segundo a classificação da American Society of Anesthesiologist (ASA), igual a ASA I ou ASA II; não ter realizado cirurgia prévia, no mínimo, nos dois meses anteriores à atual cirurgia; ser capaz de ler e escrever; estar com comorbidades compensadas. 


\section{Instrumentos}

A Escala de Fadiga de Piper - Revisada (PIPER) foi utilizada para avaliação de fadiga ${ }^{(10-11)}$. É um instrumento multidimensional, composto por 22 itens distribuídos em três dimensões, a dimensão comportamental física avalia componentes da capacidade funcional que podem ser prejudicados pela fadiga. A dimensão afetiva que busca identificar a interpretação ou o significado atribuído à fadiga. E a dimensão sensorial/psicológica que reúne componentes de auto-percepção, emocionais e cognitivos. Cada item é apresentado em escala numérica (0 a 10) e o escore total e os escores das dimensões são representados entre zero e 10, sendo que escores mais altos indicam maior fadiga. Os pacientes foram considerados fatigados quando apresentaram escore diferente de zero na escala de PIPER e, com base no escore total a fadiga foi classificada em leve (escore <4), moderada (escore $\geq 4$ e $<7$ ) e intensa (escore $\geq 7$ ).

A Escala de Estresse Percebido (EEP) foi utilizada para a avaliação da percepção de estresse. A mesma contém 14 questões que verificam o quão imprevisível, incontrolável e sobrecarregado os respondentes avaliam suas vidas. Cada item apresenta opções de resposta que variam de zero a quatro, sendo o zero igual a nunca e o quatro, sempre. O total da escala varia entre zero e 56, e quanto maior o escore, maior o estresse. Este instrumento encontra-se validado para uso no Brasil(12).

A Escala Hospitalar de Ansiedade e Depressão (HADS) foi utilizada para identificar sinais e sintomas comumente relacionados à ansiedade e à depressão. É um instrumento composto por 14 itens, sendo sete para avaliação de ansiedade e sete para a de depressão. Cada item é graduado de zero a três e os escores são calculados independentemente para cada distúrbio, variando entre zero e 21. Para esse estudo adotamos um ponto de corte 29. Esse instrumento está validado para uso no Brasil em pacientes submetidos à cirurgia(13).

A Escala Numérica de Dor foi utilizada para mensurar a intensidade da dor. É uma escala apresentada em uma linha horizontal, com 100mm de extensão, com âncoras nas extremidades: 0 (zero) = sem dor e 10 (dez) = pior dor imaginável. A Escala Numérica de Sono foi utilizada para avaliação de prejuízo de sono. É apresentada em uma linha horizontal, com 100mm de extensão, com âncoras nas extremidades: 0 (zero) = sem prejuízo de sono e 10 (dez) = sono totalmente prejudicado. A Escala de Karnofsky (KPS) foi utilizada para avaliação do grau de

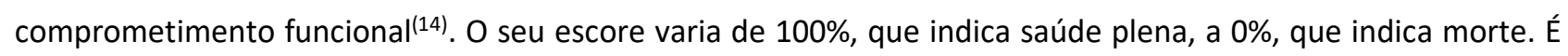
um instrumento de amplo uso na medicina brasileira, utilizado para avaliar capacidade do paciente em realizar as atividades de vida diárias e a necessidade de hospitalização diante dos sinais e sintomas da doença. Ele também é utilizado para comparar a funcionalidade em pacientes recebendo diferentes terapias, inclusive auxiliando no estabelecimento do prognóstico.

Além disso, foram analisados dados clínicos do paciente como tabagismo, comorbidades, tratamentos antineoplásicos neoadjuvantes e cirurgias prévias.

\section{Processo de coleta de dados}

Este estudo é parte da pesquisa intitulada "Fadiga perioperatória: comparação entre pacientes com e sem câncer", aprovado pelos Comitês de Ética em Pesquisa dos locais das instituições envolvidas, protocolo no 532.145 e 608.861-0. 
Os participantes da pesquisa foram recrutados durante visitas realizadas por enfermeiros e acadêmicos de enfermagem treinados, às unidades de internação buscando pacientes admitidos para o tratamento cirúrgico. Aqueles pacientes que atenderam aos critérios de inclusão, de 36 até duas horas antes da cirurgia, foram convidados para participar da pesquisa. Após leitura, esclarecimento de dúvidas e assinatura do Termo de Consentimento Livre e Esclarecido (TCLE), deu-se início à coleta de dados. Os instrumentos foram preenchidos por meio de entrevista, exame clínico e consulta ao prontuário.

\section{Análise dos dados}

Os dados foram analisados no programa estatístico SPSS (Statistical Package for Social Science) e foi adotado o nível de significância de $5 \%$. As variáveis categóricas foram descritas em frequência absoluta e relativa, e as variáveis contínuas foram expressas em médias, desvios padrão, valor mínimo e máximo. Para verificar a normalidade dos dados foi utilizado o teste de Kolmogorov-Smirnov. O teste qui-quadrado $\left(\mathrm{X}^{2}\right)$ de Pearson e teste de razão de verossimilhança foram utilizados para verificar a associação entre as variáveis categóricas. O teste tStudent e o teste Mann-Whitney foram utilizados para comparar as variáveis contínuas dos pacientes com e sem fadiga. A ANOVA oneway avaliou se os escores médios das dimensões da escala de PIPER eram iguais.

\section{RESULTADOS}

\section{Características sociodemográficas}

A idade média foi 51,2 anos, $44(37,6 \%)$ pacientes tinham até seis anos de estudo e 60 (51,3\%) referiram renda per capita inferior ao valor de um salário mínimo vigente. A maioria das pacientes foi do sexo feminino ( $n=90 ; 76,9 \%)$, vivia com companheiro $(n=77 ; 65,8 \%)$, tinha cor de pele parda ( $n=68 ; 58,1 \%)$, não era fumante ( $n=105 ; 89,7 \%)$ e não havia realizado nenhum tratamento prévio para o câncer ( $n=83 ; 70,9 \%$ ). Não houve diferença significativa entre a fadiga e as variáveis sociodemográficas investigadas (Tabela 1). 
Tabela 1: Distribuição dos pacientes com e sem fadiga segundo características sociodemográficas e clínicas. Goiânia, GO, Brasil, 2014-2015.

\begin{tabular}{|c|c|c|c|c|c|}
\hline \multirow{2}{*}{ Variáveis } & \multicolumn{2}{|c|}{ Com Fadiga $(n=30)$} & \multicolumn{2}{|c|}{ Sem Fadiga ( $n=87$ ) } & \multirow{2}{*}{$\mathbf{p}$} \\
\hline & $\mathrm{n}$ & $\%$ & $\mathbf{N}$ & $\%$ & \\
\hline \multicolumn{6}{|l|}{ Idade } \\
\hline Média (DP); min-máx & \multicolumn{2}{|c|}{$49,1(14,8) ; 21,0-88,0$} & \multicolumn{2}{|c|}{$51,9(15,0) ; 18,0-87,0$} & $0,740^{c}$ \\
\hline \multicolumn{6}{|l|}{ Renda per capita } \\
\hline Média (DP); min-máx & \multicolumn{2}{|c|}{$582,2(249,8) ; 233,3-1250$} & \multicolumn{2}{|c|}{$653,9(312,7) ; 140-3000$} & $0,987^{d}$ \\
\hline \multicolumn{6}{|l|}{ Escolaridade (anos) } \\
\hline Média (DP); min-máx & \multicolumn{2}{|c|}{8,$9 ;(3,8) ; 1,0 ; 160$} & \multicolumn{2}{|c|}{$8,4(4,1) ; 1,0 ; 16,0$} & $0,851^{d}$ \\
\hline \multicolumn{6}{|l|}{ Sexo: } \\
\hline Feminino & 26 & 86,7 & 64 & 73,6 & \multirow[t]{2}{*}{$0,125^{a}$} \\
\hline Masculino & 4 & 13,3 & 23 & 26,4 & \\
\hline \multicolumn{6}{|l|}{ Com companheiro } \\
\hline Sim & 17 & 56,7 & 60 & 69 & \multirow[t]{2}{*}{$0,221^{b}$} \\
\hline Não & 13 & 43,3 & 27 & 31 & \\
\hline \multicolumn{6}{|l|}{ Cor da pele: } \\
\hline Branco & 11 & 36,7 & 28 & 32,2 & \multirow[t]{4}{*}{$0,721^{\mathrm{a}}$} \\
\hline Preto & 2 & 6,7 & 6 & 6,9 & \\
\hline Pardo & 17 & 56,7 & 51 & 58,6 & \\
\hline Indígena & - & & 2 & 2,3 & \\
\hline \multicolumn{6}{|l|}{ Tabagismo } \\
\hline Não fumante & 26 & 86,7 & 79 & 90,8 & \multirow[t]{3}{*}{$0,535^{\mathrm{a}}$} \\
\hline Exfumante & 0 & 0 & 1 & 1,1 & \\
\hline Fumantes & 4 & 13,3 & 7 & 8 & \\
\hline \multicolumn{6}{|l|}{ Realizou cirurgia } \\
\hline Sim & 4 & 13,3 & 19 & 21,8 & \multirow[t]{2}{*}{$0,284^{a}$} \\
\hline Não & 26 & 86,7 & 67 & 77 & \\
\hline \multicolumn{6}{|l|}{ Quimioterapia neo } \\
\hline $\operatorname{Sim}$ & 3 & 10 & 16 & 18,4 & \multirow[t]{2}{*}{$0,262^{a}$} \\
\hline Não & 27 & 90 & 71 & 81,6 & \\
\hline \multicolumn{6}{|l|}{ Radioterapia neo } \\
\hline $\operatorname{Sim}$ & 1 & 3,3 & 9 & 10,3 & \multirow[t]{2}{*}{$0,196^{a}$} \\
\hline Não & 29 & 96,7 & 78 & 89,7 & \\
\hline
\end{tabular}

a teste de razão de verossimilhança; ${ }^{b}$ qui-quadrado de Pearson ; teste T-student para amostras independentes; ${ }^{d}$ teste Mann-Whitney; * Alguns dados foram deixados em branco pelos participantes e, por isso, a soma não atinge o total (100\%).

\section{Fadiga}

A prevalência de fadiga entre os pacientes em pré-operatório de cirurgia oncológica foi de $25,6 \%(n=30)$, sendo que $15,3 \%(n=18)$ apresentaram fadiga intensa, 9,4\% ( $n=11)$ fadiga moderada, e 0,9\% ( $n=1)$, fadiga leve.

A partir da avaliação das dimensões da escala PIPER, o escore médio da dimensão de fadiga afetiva foi significativamente maior que os escores médios das dimensões comportamental, sensorial/cognitiva/emocional (Tabela 2).

Tabela 2: Intensidade de fadiga segundo os domínios da Escala de Piper (n=30). Goiânia, GO, Brasil, 2015.

\begin{tabular}{cccc}
\hline & Dimensão comportamental & Dimensão afetiva & Dimensão sensorial/psicológica \\
\hline Média (DP) & $6,3(2,2)$ & $8,1(1,4)^{*}$ & $5,6(1,6)$ \\
Min - Max & $1,8-10,0$ & $5,4-10,0$ & $1,8-9,6$ \\
IC** 95\% & $5,4-7,1$ & $7,6-8,6$ & $5,0-6,2$ \\
\hline
\end{tabular}

ANOVA oneway; ${ }^{*} \mathrm{p}<0,05 ;{ }^{* *} \mathrm{IC}=$ Intervalo de confiança.

Os resultados dos testes de associação entre fadiga e as variáveis clínicas mostraram que fadiga se associou significativamente à ansiedade, à depressão, ao estresse, à dor, ao sono, e à performance status (Tabela 3). 
Tabela 3: Associação entre fadiga (escala de PIPER) e as variáveis ansiedade, depressão, estresse, dor, sono e performance status. Goiânia, GO, Brasil, 2015

\begin{tabular}{|c|c|c|c|c|}
\hline \multirow{2}{*}{\multicolumn{2}{|c|}{ Instrumentos }} & \multirow{2}{*}{$\begin{array}{c}\text { Com fadiga } \\
\mathrm{n}(\%)\end{array}$} & \multirow{2}{*}{$\begin{array}{c}\text { Sem fadiga } \\
\mathbf{n}(\%)\end{array}$} & \multirow{2}{*}{$\mathbf{p}$} \\
\hline & & & & \\
\hline \multirow{2}{*}{ HADS A } & Com ansiedade & $20(66,7)$ & $45(51,7)$ & 0,006 \\
\hline & Sem ansiedade & $10(33,3)$ & $42(48,3)$ & \\
\hline \multirow{2}{*}{ HADS D } & Com depressão & $17(56,7)$ & $21(24,1)$ & 0,004 \\
\hline & Sem depressão & $13(43,3)$ & $66(75,9)$ & \\
\hline \multirow{2}{*}{ EEP } & Alta percepção de estresse & $27(90,0)$ & $60(69,0)$ & 0,015 \\
\hline & Baixa percepção de estresse & $3(10,0)$ & $27(31,0)$ & \\
\hline \multirow{2}{*}{ Dor } & Com dor & $23(76,7)$ & $44(50,6)$ & 0,004 \\
\hline & Sem dor & $6(20,0)$ & $44(50,6)$ & \\
\hline \multirow{2}{*}{ Sono } & Com prejuízo & $26(86,7)$ & $51(58,6)$ & $<0,001$ \\
\hline & Sem prejuízo & $6(20,0)$ & $44(50,6)$ & \\
\hline \multirow{2}{*}{ KPS } & $100 \%-90 \%$ & $10(33,3)$ & $71(81,6)$ & $<0,001$ \\
\hline & $\leq 80 \%$ & $20(66,7)$ & $15(17,2)$ & \\
\hline
\end{tabular}

HADS A: Escala Hospitalar de Ansiedade e Depressão - Ansiedade; HADS D: Escala Hospitalar de Ansiedade e Depressão - Depressão; EEP: Escala de Estresse Percebido; KPS: Karnofsky Performance Status.

\section{DISCUSSÃO}

\section{Características sociodemográficos}

Os resultados não mostraram associação entre fadiga e as variáveis sociodemográficas. Por um lado, esses resultados corroboram com os achados de outros autores, reforçando a hipótese de que a fadiga pré-operatória não está associada a sexo, idade, viver com companheiro, cor da pele, renda per capita ${ }^{(15)}$. Por outro, alguns estudos apresentaram resultados diferentes. Em mulheres com câncer de mama em pré-operatório, fadiga se associou a escolaridade e ter companheiro ${ }^{(16)}$. Outro estudo, também envolvendo mulheres com câncer de mama em pré-operatório, identificou a associação entre fadiga e estado civil, idade, filhos, emprego ${ }^{(17)}$, ou seja, mulheres com pouca escolaridade, solteiras, mais velhas, com filhos e sem emprego, apresentavam mais fadiga no préoperatório.

Embora muitos artigos apontem a associação entre fadiga e quimioterapia, radioterapia e tabagismo(7,18), nossos resultados não mostraram tal associação. O mesmo foi encontrado em outras pesquisas, que mostraram que quimioterapia neoadjuvante não influenciou a intensidade dos sintomas, comprovando que terapias antineoplásicas neoadjuvantes não predisseram fadiga(19-20).

\section{Fadiga pré-operatória}

Os resultados indicaram a prevalência de fadiga pré-operatória igual a $25,6 \%$. Alguns estudos também encontraram uma prevalência semelhante. Uma pesquisa realizada em pacientes com câncer colorretal, de ambos os sexos, a fadiga foi relatada por $22 \%$ dos pacientes antes da cirurgia ${ }^{(21)}$. Já em pacientes com câncer de próstata, a prevalência de fadiga foi igual a 14,3\%; em pacientes com câncer de mama, foi igual a 20,3\%; e, em pacientes com cânceres do trato gastrointestinal, fadiga foi referida por $28,1 \%$ dos pesquisados ${ }^{(22)}$.

Dos pacientes fatigados no pré-operatório, a imensa maioria referiu intensidade moderada a intensa. Esse resultado indica que a fadiga desses pacientes cirúrgicos é clinicamente significativa, demandando medidas para seu controle. Ao examinar a literatura, observou-se que alguns estudos apresentaram resultados semelhantes ao 
presente estudo, e que os pacientes estavam com fadiga moderada antes do tratamento cirúrgico ${ }^{(8-9,23)}$. Esses estudos foram realizados com pacientes de ambos os sexos, com a idade média de 58 anos, em pré-operatório para câncer de trato digestivo alto, pulmão, mama e ginecológico. Um estudo avaliou pacientes com câncer ósseo ou de tecidos moles e encontrou uma prevalência de $29,7 \%$ e desses, $35 \%$ tinham fadiga intensa antes da cirurgia ${ }^{(3)}$.

Em relação aos domínios da Escala de Fadiga, a dimensão afetiva obteve o maior escore médio. Destacando que a percepção quanto aos sintomas no período de espera antes da cirurgia podem ficar mais evidente e ser interpretado como negativa, tornando a fadiga mais desagradável, inaceitável, destruidora e anormal, já que na maioria das vezes, essa situação nunca foi experimentada pelo paciente ${ }^{(10)}$. Encontramos apenas um estudo que reportou a dimensão da fadiga, este foi realizado em mulheres com câncer de mama, e o domínio que obteve o maior escore foi o físico, seguido do afetivo e cognitivo ${ }^{(20)}$. A dimensão afetiva, não foi a que obteve o maior escore nesse caso, mas teve uma presença significativa, confirmando seu prejuízo ao paciente como encontrado em nosso estudo. Apesar dos estudos referirem a importância de instrumentos multidimensionais, ainda são poucos que avaliam as dimensões da fadiga, dificultando a comparação dos resultados.

Frequentemente, o paciente no pré-operatório, recém-diagnosticado, ainda não foi submetido a outras fontes geradoras de fadiga, além do próprio câncer, das emoções ocasionados pelo diagnóstico e pela expectativa do procedimento cirúrgico. No presente estudo, todos os fatores clínicos avaliados (ansiedade, depressão, estresse, dor, sono e performance) se associaram à fadiga. Resultados semelhantes foram descritos anteriormente, onde mostram que os impactos desses fatores parecem intensificar a manifestação da fadiga ${ }^{(3,24)}$. Sendo imprescindível que, principalmente nesses pacientes, os profissionais investiguem e avaliem a fadiga, de forma a evitar que a mesma seja sub-relatada, sub-diagnosticada e sub-tratada, provocando maiores prejuízos na recuperação do paciente ${ }^{(3)}$.

Atualmente, muitas publicações reportam cluster de sintomas que incluem fadiga ${ }^{(18,25)}$. Estudos futuros, com amostras maiores, poderão confirmar os resultados encontrados no presente estudo e, também, buscar clusters de sintomas para pacientes em pré-operatório de cirurgia oncológica.

\section{CONCLUSÕES E IMPLICAÇÕES PARA A PESQUISA E PRÁTICA CLÍNICA}

Esse estudo, sugere que uma porcentagem significativa dos pacientes com câncer, no pré-operatório, apresenta-se fatigada de modo clinicamente significativo, visto a intensidade do sintoma. A prevalência de fadiga entre os pacientes em pré-operatório de cirurgia oncológica foi de $25,6 \%$. A dimensão afetiva de fadiga apresentou escores significativamente mais altos que das demais dimensões e, sabendo disto, o profissional deve atuar na interpretação do sintoma referido pelos pacientes, por exemplo, orientando o enfrentamento focado no problema e não nas emoções.

Os resultados desta pesquisa mostraram associação entre a fadiga pré-operatória e ansiedade, depressão, estresse e alterações de sono. De certa forma, esses sintomas são esperados e considerados normais em pessoas que estão prestes a serem submetidos à cirurgia. Por haver expectativas de que esses sintomas estejam presentes, infelizmente, quando presentes, não são adequadamente avaliados e manejados. Outros fatores como a dor e piora da funcionalidade, também se mostraram associados à fadiga. É provável que estes dois fatores estejam 
relacionados ao câncer, e não à expectativa do procedimento cirúrgico, apesar de se saber que a dor é também multidimensional e influenciada pelas emoções.

A assistência de enfermagem ao paciente em pré-operatório é um desafio. O enfermeiro, junto à equipe multiprofissional pode auxiliar na detecção desses sintomas, em especial da fadiga, por meio de instrumentos válidos e confiáveis. A identificação da fadiga e sua avaliação por meio de instrumento multidimensional fornecem evidências para a definição de intervenções para a fadiga.

\section{Agradecimentos}

Essa pesquisa foi financiada pelo MCTI/CNPq/MEC/Capes (Ação Transversal no/2011 Casadinho/PROCAD).

\section{REFERÊNCIAS}

1. Schroeder D, Hill GL. Predicting postoperative fatigue: importance of preoperative factors. World J Surg [Internet]. 1993 [acesso em: 05 set. 2018];17(2):226-31. Disponível em: https://doi.org/10.1007/BF01658931.

2. Gorini MIPC, Silva POS, Chaves PL, Ercole JP, Cardoso BC. Registro do diagnóstico de enfermagem fadiga em prontuários de pacientes oncológicos. Acta paul. enferm. [Internet]. 2010 [acesso em: 05 set. 2018]; 23(3):356-8. Disponível em: https://doi.org/10.1590/S0103-21002010000300007.

3. Van der Geest ICM, Knoop H, Veth RPH, Schreuder HWB, Bleijenberg G. High fatigue scores before and after surgical treatment of bone and soft tissue tumors. Exp Ther Med [Internet]. 2013 [acesso em: 05 set. 2018];5(1):205-8. Disponível em: https://doi.org/10.3892/etm.2012.786.

4. Bulechek GM, Butcher HK, Dochterman JM, Wagner CM. Nursing Interventions Classifiction (NIC). $6^{\text {th }}$ ed. St Louis: ElsevierMosby; 2013.

5. Mota DDCF, Cruz DALM, Pimenta CAM. Fadiga: uma análise do conceito. Acta paul. enferm. [Internet]. 2005 [acesso em: 05 set. 2018];18(3):285-93. Disponível em: https://doi.org/10.1590/S0103-21002005000300009.

6. Herdman TH. Diagnósticos de enfermagem da NANDA: definições e classificação 2012-2014. Porto Alegre: ARTMED; 2013.

7. Oliveira MM, Oliveira GF, Souza-Talarico JN, Mota DDCFM. Surgical Oncology: Evolution of Postoperative Fatigue and Factores Related to Its Severity. Clin J Oncol Nurs [Internet]. 2016 [acesso em: 05 set. 2018];20(1):E-3-8. Disponível em:

https://doi.org/10.1188/16.CJON.E3-E8.

8. Barbour AP, Lagergren P, Hughes R, Alderson D, Barham CP, Blazeby JM. Health-related quality of life among patients with adenocarcinoma of the gastro-oesophageal junction treated by gastrectomy or oesophagectomy. Br J Surg [Internet]. 2008 [acesso em: 05 set. 2018];95(1):80-4. Disponível em: https://doi.org/10.1002/bjs.5912.

9. Schrepf A, Clevenger L, Christensen D, DeGeest K, Bender D, Ahmed A, et al. Cortisol and inflammatory processes in ovarian cancer patients following primary treatment: relationships with depression, fatigue, and disability. Brain Behav Immun [Internet]. 2013 [acesso em: 05 set. 2018];30 Suppl:S126-34. Disponível em: https://doi.org/10.1016/j.bbi.2012.07.022.

10. Piper BF, Dibble SL, Dodd MJ, Weiss MC, Slaughter RE, Paul SM. The revised Piper Fatigue Scale: psychometric evaluation in women with breast cancer. Oncol Nurs Forum. 1998 May;25(4):677-84.

11. Mota DDCF, Pimenta CAM, Piper BF. Fatigue in Brazilian cancer patients, caregivers and nursing students: a psychometric validation study of the Piper Fatigue Scale-Revised. Support Care Cancer [Internet]. 2009 [acesso em: 05 set. 2018];17(6):645-52. Disponível em: https://doi.org/10.1007/s00520-008-0518-x.

12. Luft CB, Sanches SO, Mazo GZ, Andrade A. Versão brasileira da escala de estresse percebido: tradução e validação para idosos. Rev Saude Publica [Internet]. 2007 [acesso em: 05 set. 2018];41(4):606-615. Disponível em: https://doi.org/10.1590/S0034$\underline{89102007000400015 .}$

\section{Retratado}

13. Marcolino JAM, Mathias LAST, Piccinini Filho L, Guaratini AA, Suzuki FM, Alli LAC. Escala Hospitalar de Ansiedade e Depressão: Estudo da validade de critério e da confiabilidade com pacientes no pré-operatório. Rev Bras Anestesiol [Internet]. 2007 [acesso em: 05 set. 2018];57(1):52-62. Disponível em: http://www.scielo.br/pdf/\%0D/rba/v57n1/06.pdf.

14. Karnofsky DA, Burchenal JH. The Clinical Evaluation of Chemotherapeutic Agents. In: MacLeod CM, editor. Evaluation of Chemotherapeutic Agents. New York: Columbia Univ. Press; 1949. p. 196.

15. Lockefeer JP, De Vries J. What is the relationship between trait anxiety and depressive symptoms, fatigue, and low sleep quality following breast cancer surgery? Psychooncology [Internet]. 2013 [acesso em: 05 set. 2018];22(5):1127-33. Disponível em:

https://doi.org/10.1002/pon.3115. 
16. Huang HP, Chen ML, Liang J, Miaskowski C. Changes in and predictors of severity of fatigue in women with breast cancer: A longitudinal study. Int J Nurs Stud [Internet]. 2014 [acesso em: 05 set. 2018];51(4):582-92. Disponível em:

https://doi.org/10.1016/j.ijnurstu.2013.09.003.

17. Michielsen HJ, Van der Steeg AFW, Roukema JA, De Vries J. Personality and fatigue in patients with benign or malignant breast disease. Support Care Cancer [Internet]. 2007 [acesso em: 05 set. 2018];15(9):1067-73. Disponível em:

https://doi.org/10.1007/s00520-007-0222-2.

18. Thomas BC, Waller A, Malhi RL, Fung T, Carlson LE, Groff SL, et al. A longitudinal analysis of symptom clusters in cancer patients and their sociodemographic predictors. J Pain Symptom Manage [Internet]. 2014 [acesso em: 05 set. 2018];47(3):566-78.

Disponível em: https://doi.org/10.1016/j.jpainsymman.2013.04.007.

19. Sarna L, Cooley ME, Brown JK, Chernecky C, Elashoff D, Kotlerman J. Symptom severity 1 to 4 months after thoracotomy for lung cancer. Am J Crit Care [Internet]. 2008 [acesso em: 05 set. 2018];17(5):455-67. Disponível em:

http://ajcc.aacnjournals.org/content/17/5/455.long.

20. Haghighat S, Akbari ME, Holakouei K, Rahimi A, Montazeri A. Factors predicting fatigue in breast cancer patients. Support Care Cancer [Internet]. 2003 [acesso em: 05 set. 2018];11(8):533-8. Disponível em: https://doi.org/10.1007/s00520-003-0473-5.

21. Tsunoda A, Nakao K, Tsunoda Y, Watanabe M, Matsui N. Health-related quality of life of colorectal cancer patients receiving oral UFT plus leucovorin compared with those with surgery alone. Int J Clin Oncol [Internet]. 2010 [acesso em: 05 set.

2018];15(2):153-60. Disponível em: https://doi.org/10.1007/s10147-010-0035-z.

22. Goedendorp MM, Gielissen MFM, Verhagen CAH, Peters MEJW, Bleijenberg G. Severe fatigue and related factors in cancer patients before the initiation of treatment. Br J Cancer [Internet]. 2008 [acesso em: 05 set. 2018];99(9):1408-14. Disponível em: https://doi.org/10.1038/sj.bjc.6604739.

23. Montgomery GH, Schnur JB, Erblich J, Diefenbach MA, Bovbjerg DH. Presurgery psychological factors predict pain, nausea, and fatigue one week after breast cancer surgery. J Pain Symptom Manage [Internet]. 2010 [acesso em: 05 set. 2018];39(6):1043-52.

Disponível em: https://doi.org/10.1016/j.jpainsymman.2009.11.318.

24. Denieffe S, Cowman S, Gooney M. Symptoms, clusters and quality of life prior to surgery for breast cancer. J Clin Nurs [Internet]. 2014 [acesso em: 05 set. 2018];23(17-18):2491-502. Disponível em: https://doi.org/10.1111/jocn.12430. 25. Trudel-Fitzgerald C, Savard J, Ivers H. Longitudinal changes in clusters of cancer patients over an 18-month period. Health Psychol [Internet]. 2014 [acesso em: 05 set. 2018];33(9):1012-22. Disponível em: https://doi.org/10.1037/a0033497. 